\title{
On the semantics of bipolarity and fuzziness
}

\author{
J. Tinguaro Rodríguez a , Camilo A. Franco, Javier Montero
}

\begin{abstract}
This paper analyzes the relationship between fuzziness and bipolarity, notions which were devised to address different kinds of uncertainty: linguistic imprecision, in the former, and knowledge relevance and character or polarity, in the latter. Although different types of fuzziness and bipolarity have been defined, these relations are not always clear. This paper proposes the use of four-valued extensions to provide a formal method to rigorously define and compare the semantics and logical structure of diverse combinations of fuzziness and bipolarity types. As a result, this paper claims that these notions and their different types are independent and not semantically equivalent despite its possible formal equivalence.
\end{abstract}

\section{Introduction}

Fuzziness [39] and bipolarity [11] are two independent but complementary notions originally (and separately) devised to face the mathematical modelling of different features of natural languages and human reasoning. Though their influence (more than considerable in fields as decision theory [14,17,26,29] or machine learning [19]) has spread separately, in the last few decades both notions have started to appear together in many developments on these and other fields (see for instance [4,6,13,15,30, $31,38]$ ), which comes to show its high relevance as a topic of research inside soft computing [20] and logics [36].

However, the relationships (and differences) between fuzziness and bipolarity are not always clear. In order to introduce our point, let us remind that, on one hand, fuzziness is concerned with the imprecision inherent to natural languages: many relevant predicates (i.e. words) $P$, as good or young, have ill-defined boundaries, and uncertainty arises regarding whether objects $x$ of a universe of discourse $X$ (e.g. decision al ternatives or ages of customers) fulfil them or not.

Faculty of Mathematics, Complutense University of Madrid, Plaza de Ciencias 328040 Madrid, Spain a: jtrodrig@mat.ucm.es 
On the other hand, bipolarity is concerned with the character (or polarity) and relevance of information: it has become clear (see [7,25,28]) that human reasoning tends to analyze reality (e.g. a decision to be taken $[23,29]$ ) by checking separately both the positive and negative sides of the available information (e.g. an alternative could be good for certain criteria and bad for other set of criteria) in order to acquire a more expressive and relevant knowledge. Thus, reality is judged in terms of pairs of poles of reference $P / Q$, as false/true or good/bad, which organize and give relevance to the available information.

Moreover, different types of fuzziness [40] and bipolarity [12] have been studied and defined. While usual (type-1) fuzziness (F1) measures linguistic imprecision in a precise way (assigning a gradable but precise truth-value $\mu_{P}(x) \in[0,1]$ to the proposition " $x$ fulfils $P$ ", thus modelling $P$ as a fuzzy set), type-2 fuzziness (F2) enables such an imprecision to be measured imprecisely (since it assigns a fuzzy set of the truth scale $[0,1]$ to $\left." x \in P^{\prime \prime}\right)$.

Similarly, while type-1 bipolarity (B1) relies on the idea that negative information is just the negation or complementation of the positive one, type-2 bipolarity (B2) allows the relation between poles to be not so simple (for example bad $\neq$ not good), and thus evaluating the pair $\left(\mu_{P}(x), \mu_{Q}(x)\right)$ could be necessary in order to capture all relevant information.

Notice that, as they try to address different kinds of uncertainty, fuzziness and bipolarity seem to be not necessarily related or interlinked: in principle a B2 formalism could be either an F1 or F2 (or even crisp!) model, and an F2 framework could be associated to either a B1 or B2 setting. Nevertheless, a commonly-used instance of type2 fuzziness, interval valued fuzzy sets (IVFS, see [18]), actually devised as B1 objects, has been shown (see [8,9]) to be in certain sense equivalent to Atanassov fuzzy sets (AFS, see [1]), which however were originally devised as F1 and B2 objects. In fact, as a consequence of this formal equivalence, a bitter dispute (see [10] and [3]) raised between Atanassov and his followers, on one side, and an important part of the fuzzy community, on the other, about the exact meaning of AFS and their real relevance in the context of bipolarity.

The main objective of this paper is to shed some light on the relations between fuzziness and bipolarity from a different perspective, and try to lead the referred differences between Atanassov and his detractors, apparently not totally solved, to a definitive solution. For this aim, the notion of four-valued extension (that clearly resembles that of preference structure) is used in order to rigorously define and compare the semantics and underlying logical structure of each possible combination of fuzziness and bipolarity types 1 and 2. This will allow us to separate and distinguish IVFS from AFS in a practical way, and will enable us to show the independency of fuzziness and bipolarity. AFS in a practical way, and will enable us to show the independency of fuzziness and bipolarity. AFS in a practical way, and will enable us to show the independency of fuzziness and bipolarity. 
On the semantics of bipolarity and fuzziness

This paper is organized as follows: the notions of type- 1 and type-2 fuzziness are revised in Section 2, and those of type-1 and type-2 bipolarity will be revised in Section 3. Four-valued extensions are introduced and applied to the four possible combinations of bipolarity and fuzziness types in Section 4. Finally, some conclusions are shed in Section 5.

\section{Type-1 and type-2 fuzziness}

Since the first proposal of L.A. Zadeh in the middle-sixties of the last century (see [39]), fuzzy set theory and fuzzy logic have enabled an increasingly sophisticated mathematical treatment of the imprecision inherent to natural languages. As said above, the imprecision of a predicate $P$ entails uncertainty about whether objects $x$ of a universe of discourse $X$ verify it or not. Fuzzy logic addresses this uncertainty by allowing the truth of the proposition " $x$ verifies $P$ " (i.e. " $x \in P$ ") to be evaluated in the interval $[0,1]$ (rather than in the classical, binary valuation space $\{0,1\}$ ). Therefore, the crisp index function of $P$ is generalized into a membership function $\mu_{P}: X \rightarrow[0,1]$ that specifies the degree up to which each object $x$ verifies $P$. This enables objects to partially fulfil an imprecise predicate or, in other words, the semantics (or use, see [34]) of $P$ on $X$ is modeled as a (type-1) fuzzy set (T1FS) $P=\left\{\left(x, \mu_{P}(x)\right) \mid x \in X\right\}$.

Also, in response to some criticism raised about the possibility of obtaining totally precise membership-degrees $\mu_{P}(x) \in[0,1]$, L.A Zadeh introduced in [40] the notion of type-n fuzziness by allowing to measure the truth of " $x \in P$ " by means of a type- $n-1$ fuzzy set of $[0,1]$. Particularly, if $F([0,1])$ denotes the set of all type-1 fuzzy sets of $[0,1]$, a type-2 fuzzy set (T2FS) $P$ is associated with a membership function $\psi_{P}: X \rightarrow F([0,1])$, in such a way that $\psi_{P}(x):[0,1] \rightarrow[0,1]$ expresses the plausibility-degree of the proposition " $x$ verifies $P$ with truth-degree $\mu$ " for each truth-degree $\mu \in[0,1]$. Therefore, imprecision in the measurement of type- 1 truth degrees $\mu_{P}(x)$ is allowed and, in general, higher types of fuzziness enable further imprecision to be introduced in truth degrees.

Perhaps the simplest and most used instance of type-2 fuzzy sets are intervalvalued fuzzy sets (IVFS, see [18]), which assign to each object $x$ and predicate $P$ an interval $\left[\mu_{L}(x), \mu_{U}(x)\right]_{P}$ as (equally and totally) plausible values of the truth degree $\mu_{P}(x)$. Therefore, the valuation space of IVFS is the set

$$
L^{I}=\{[a, b] \subseteq[0,1] \mid a \leq b\} \subset F([0,1]) .
$$

Also, the wider an interval $\left[\mu_{L}(x), \mu_{U}(x)\right]$ is, the bigger the uncertainty associated to it, where its length $u(x)=\mu_{U}(x)-\mu_{L}(x)$ is usually taken as the degree of uncer- 
tainty inherent to such an evaluation. Thus, $u(x)=0$ if there is not uncertainty about the degree up to which $x$ verifies $P$, i.e. if $\mu_{L}(x)=\mu_{P}(x)=\mu_{U}(x)$.

Classic logical connectives such as not, and, or can be generalized by means of different fuzzy operators. The usual negation [22] for type-1 fuzzy sets is given by $n(\mu)=1-\mu$, so that $\mu_{\neg P}(x)=n\left(\mu_{P}(x)\right)$, with $\neg P=$ not- $P$. Notice also that the usual negation defined over IVFS is $n_{L^{I}}\left(\left[\mu_{L}(x), \mu_{U}(x)\right]_{P}\right)=\left[1-\mu_{U}(x), 1-\mu_{L}(x)\right]_{-P}$.

Also, t-norms and t-conorms $[22,33]$ are usually taken as operators for conjunction and disjunction, respectively. Both connectives are related through the negation $n$, so that if $T$ is a t-norm, then $S\left(\mu_{P}, \mu_{Q}\right)=n\left[T\left(n\left(\mu_{P}\right), n\left(\mu_{Q}\right)\right)\right]$ is a t-conorm. For T1FS, common examples of so related operators are the Lukasiewicz t-norm and t-conorm, respectively given, for $a, b \in[0,1]$, by $a$ e $b=\max \{a+b-1,0\}, a \oplus b=\min \{a+b, 1\}$, or the minimum $a \wedge b=\min \{a, b\}$ and the maximum $a \vee b=\max \{a, b\}$. For IVFS, these operators are extended as follows:

$$
\begin{gathered}
{[a, b] \mathrm{e}_{I}[c, d]=[a \mathrm{e} c, b \mathrm{e} d],[a, b] \oplus_{I}[c, d]=[a \oplus c, b \oplus d],} \\
{[a, b] \wedge_{I}[c, d]=[a \wedge c, b \wedge d],[a, b] \vee_{I}[c, d]=[a \vee c, b \vee d] .}
\end{gathered}
$$

Notice that fuzzy logic seems to underestimate the notion of negative information. If any, it assumes that the falsehood of " $x \in P$ " is equal to the truth of " $x \notin P$ ", i.e. $\mu_{\neg P}(x)$, though this falsehood could be of different nature than negative information. Anyway, it is clear that fuzzy logic does not consider an extra, independent evaluation (being either a falsehood degree or a measure of negative information) together with the (precise or not) degrees $\mu_{P}(x)$ or $\psi_{P}(x)$.

Typical applications of fuzzy logic (today more or less covered below the term soft computing [20]) include, among others, intelligent control [24], decision theory [14] or machine learning [19]. Type-2 FS (specially IVFS) have also found extensive application in various fields (see for example [6]).

\section{Type-1 and type-2 bipolarity}

Although the idea of measuring independent positive and negative information has a psychological inspiration (see $[25,28]$ ) and has appeared separately and without a unitary label (and even not explicitly) in the scientific literature (specially that concerned with decision theory), quite recently the term bipolarity seems to have succeeded in becoming a widely accepted label for this rather general idea [11].

Basically, bipolarity assumes the existence of a pair of reference poles $P / Q$, as false/true or good/bad, which provide absolute landmarks that confer information its intrinsic positive and negative character. Information having neither positive nor negative character is therefore irrelevant or neutral in terms of such references. Thus, the poles $P / Q$ organize and give relevance to the available information, so that positive information for one of the poles is taken as against the other. 
On the semantics of bipolarity and fuzziness

If the relation between the poles $\mathrm{P}$ and $\mathrm{Q}$ is given by the complementation, i.e. $Q=n o t P=\neg P \quad$ (e.g. bad=not good), then a single evaluation $\mu_{P}(x) \in[0,1]$ (or $\left.\mu_{P}(x) \in L^{I}\right)$ is enough to capture all the information that is relevant in terms of the polarity $P / Q$, since then $\mu_{Q}(x)=\mu_{\neg P}(x)=1-\mu_{P}(x)$ and therefore the negative information is just the negation of the positive one. This situation is usually referred to as type-1 bipolarity (B1, see [13]), and let us remark that fuzzy sets (both F1 and F2) are usually assumed to belong to this category as exposed in last section.

However, if the relation between $P$ and $Q$ is not so simple (e.g. bad $\neq$ not good), then it is usually also necessary to evaluate $\mu_{Q}(x)$ in order to capture all the relevant information about " $x \in P$ ". Particularly, two different scales $L_{P}^{+}$and $L_{P}^{-}$can be used to evaluate, respectively, $\mu_{P}(x)$ and $\mu_{Q}(x)$, in such a way that the evidence couple $\left(\mu^{+}(x), \mu^{-}(x)\right)_{P}=\left(\mu_{P}(x), \mu_{Q}(x)\right) \in L_{P}^{+} \times L_{P}^{-}$measures the degree of positive and negative information regarding " $x \in P$ ". Besides, if the relation between the poles is assumed to be symmetric (though it is possible to remove this assumption, see [31]), then

$$
\left(\mu^{+}(x), \mu^{-}(x)\right)_{Q}=\left(\mu_{Q}(x), \mu_{P}(x)\right) \in L_{Q}^{+} \times L_{Q}^{-}=L_{P}^{-} \times L_{P}^{+} .
$$

These bivariate evidences are typical of type-2 bipolarity (B2, see [13]), and note that, as $P$ and $Q$ are not necessarily complementary, B2 models in principle admits 4 possible cases or epistemic states regarding an object $x$ and the pair $P / Q$ :

- Positive truth $t: x$ verifies $P$ but does not verify $Q$, i.e., information is positive and not negative. The extreme (or crisp) representative of this state is given by the evidence couple $\left(\mu^{+}, \mu^{-}\right)=(1,0)$;

- Negative truth (or positive falsehood) $f: x$ fulfils $Q$ and does not verify $P$, i.e., information is negative and not-positive. Its extreme pair is $\left(\mu^{+}, \mu^{-}\right)=(0,1)$;

- Irrelevance or neutrality $i$ : $x$ neither fulfils $P$ nor $Q$, and thus information is both not-positive and not-negative, so in the limit it is $\left(\mu^{+}, \mu^{-}\right)=(0,0)$;

- Conflict $k$ : $x$ simultaneously fulfil $P$ and $Q$, so information is positive and negative, as in the crisp case $\left(\mu^{+}, \mu^{-}\right)=(1,1)$.

Let us remark that the third and fourth cases are not possible in B1 frameworks (as fuzzy logic), in which $P$ and $Q$ are tightly linked through the negation. If the relationship between the poles is somehow restricted (for example, by introducing a constraint in $L^{+} \times L^{-}$), the third or the fourth case could cease to hold, but not simultaneously: if this is the case, then we are back in a B1 setting.

In relation with preference modelling and decision analysis, the notion of independent positive and negative information has also appeared in, among others, cumulative prospect theory (see [21,37]), outranking concordance-discordance ELECTRE methods (see [32]), Atanassov intuitionistic fuzzy sets (AFS, see [1]) and, more re- 
cently, in DDT logic [35]. Current applications of type-2 bipolarity along with fuzzy logic are promising and increasing (see [15,29,30,38])

It is interesting to examine in more detail the case of AFS, which assign to " $x \in P$ " both a degree of membership $\mu_{P}(x)$ and an independent degree of nonmembership $\mu_{\neg P}(x)$, such that $\mu_{P}(x), \mu_{\neg P}(x) \in[0,1]$ and $\mu_{P}(x)+\mu_{\neg P}(x) \leq 1$, i.e, $L^{*}=\left\{(x, y) \in[0,1]^{2} \mid x+y \leq 1\right\} \subset[0,1]^{2}$ is the valuation space of AFS, and notice that the same scale $L^{+}=L^{-}=[0,1]$ is used for both evaluations (though a constraint has been included). Note also that Atanassov implicitly treats a B1 framework (i.e. in which the relevant polarity is $P / \neg P$ ) as a B2 one, assuming that the truth of " $x \notin P$ " can not be obtained from that of " $x \in P^{\prime}$, thus requiring an independent evaluation $\mu_{-P}(x)=v_{P}(x)$. As it is $\mu_{P}(x)+v_{P}(x) \leq 1$, it is assumed that the disjunction of $P$ and its negation (in terms of the Lukasiewicz t-conorm) could not be a tautology, thus violating the law of the excluded middle $(P \vee-P)$, that was Atanassov's aim as he wanted its model to be intuitionistic.

However, other fuzzy structures also violate such a law without considering an independent negation (for example, by taking the maximum t-conorm $\vee$ and $\left.\mu_{\neg P}(x)=1-\mu_{P}(x)\right)$. Thus, the exact meaning of AFS is rather obscure, showing the semantics of a B1 framework while formally seeming to be a B2 one. Moreover, as Atanassov and his followers failed to give a clear definition of AFS's underlying logical and semantical structure (see [27]), no solid reasons to separate AFS from IVFS were available when both formalisms were proven $[8,9]$ to be equivalent (through the isomorphism $\Phi: L^{*} \rightarrow L^{I}$ given by

$$
\left.\Phi\left(\left\langle\mu_{P}(x), \mu_{\neg P}(x)\right\rangle\right)=\left[\mu_{P}(x), 1-\mu_{\neg P}(x)\right]\right) .
$$

This equivalence triggered a strong controversy (see $[3,10])$ between Atanassov and his followers, which thought AFS were a valid B2 model (or at least a valid intuitionistic model) different from IVFS, and an important part of the fuzzy community, which instead thought the intuitionistic meaning of AFS was not clear at all and, since IVFS were formulated some years before than AFS, the relevance of the latter as bipolar objects should be reduced due to such equivalence (despite IVFS were originally conceived as B1 objects).

In our opinion, AFS' original semantics (whatever it may be) is not of B2 type, and therefore AFS are not a really relevant landmark in the field of bipolarity. However, if $v$ is interpreted as the membership function of a polarity $Q$ such that $Q \neq \neg P$, i.e. $v_{P}(x)=\mu_{Q}(x)$, and the constraint $\mu_{P}(x)+v_{P}(x) \leq 1$ is maintained, then a B2 semantics (with a particular relation between poles $P$ and $Q$ ) is easily obtained, and our opinion is that these objects (which will be referred to as bipolar AFS: BAFS) are not semantically equivalent to IVFS. In order to support this last claim, we will formally define the semantics of B2 logical objects, and will show that semantics of BAFS (and 
On the semantics of bipolarity and fuzziness

in general that of F1 and B2 formalisms) is different from that of IVFS (in general, from that of B1 and F2 models). Such a semantical differentiation will be introduced through the notion of four-valued extension.

\section{Four-valued extensions}

Given an evidence couple $\left(\mu^{+}, \mu^{-}\right)$that evaluates the positive and negative information regarding " $x \in P$ ", how can we assess (and quantify) from such evidence whether $x$ can be identified with the positive pole $P$ (in the sense of having positive character or verifying the positive truth epistemic state $t$ ) or not? In other words, what is the exact or relevant meaning or semantics of a given evidence couple? For example, the pair $\left(\mu^{+}, \mu^{-}\right)=(1,0)$ indicates that $x$ has a fully positive character, and thus the positive truth of " $x \in P^{\prime}$ can be assumed in this case to be maximum, i.e., 1. This could lead to think that $t$ coincides with the truth of " $x \in P^{\prime \prime}$ (since $\left.\mu^{+}(x)=\mu_{P}(x)=1\right)$ or the falsehood of $Q$ (since $\neg \mu^{-}(x)=1-\mu_{Q}(x)=1$ ).

However, the pairs $(1,1)$ or $(0,0)$ do not represent neither a prevalence of the positive pole over the negative one nor the reciprocal. Rather, they make it clear that in a B2 context, the truth of " $x \in P$ " (i.e. $\mu_{P}(x)$ ) should not be directly identified with the positive truth epistemic state $t$ resulting from conjointly evaluating positive and negative information. Moreover, as in a fuzzy (F1 or F2), type-2 bipolar setting an object $x$ could be partially compatible with both poles $P$ and $Q$, the associated evidence couple $\left(\mu^{+}, \mu^{-}\right)$could be partially compatible with more than one of the epistemic states $t, f, i, k$ described in last section. Therefore, these states are also gradable or fuzzy in nature, and the notion of 4-valued extension is useful to quantify these compatibilities (and thus the semantics of the evidence couple) under some reasonable assumptions (as in preference modelling [14], where the 4 values of the preference structure are the relevant, final items of the analysis).

Thus, supposing that $L^{+}=L^{-}=L$, with each evidence couple $\left(\mu^{+}, \mu^{-}\right) \in L^{+} \times L^{-}$there is associated an evidence matrix

$$
E M=\left[\begin{array}{ll}
t\left(\mu^{+}, \mu^{-}\right) & i\left(\mu^{+}, \mu^{-}\right) \\
k\left(\mu^{+}, \mu^{-}\right) & f\left(\mu^{+}, \mu^{-}\right)
\end{array}\right],
$$

obtained through the formulae

$$
\begin{gathered}
t\left(\mu^{+}, \mu^{-}\right)=\mu^{+} \wedge \neg \mu^{-}, f\left(\mu^{+}, \mu^{-}\right)=\neg \mu^{+} \wedge \mu^{-} \\
i\left(\mu^{+}, \mu^{-}\right)=\neg \mu^{+} \mathrm{e}_{w} \neg \mu^{-}, k\left(\mu^{+}, \mu^{-}\right)=\mu^{+} \mathrm{e}_{w} \mu^{-},
\end{gathered}
$$

where $\neg \mu=n \circ \mu=1-\mu$. Note that $t, f, i, k \in L$, and therefore $E M$ assesses the degree up to which each of these four epistemic states hold in the same scale in which both $\mu^{+}$and $\mu^{-}$are measured. The 4-valued extension given in formulae (1)-(2) was first proposed for the case $L=[0,1]$ in [29] (and have been further analyzed in [36]), in 
which it is proven that it is the unique continuous t-norm-based extension simultaneously verifying that the states $i$ and $k$ are mutually exclusive (since if $i>0$ then $k=0$ and vice versa) and $t+f+i+k=1$. Notice that by exchanging the places of the Lukasiewicz t-norm e and the minimum t-norm $\wedge$, a different extension is obtained (and in fact it is used in preference modelling, see [14]), in which the last equality also holds but now $t$ and $f$ are exclusive. Nevertheless, as we shall see, this last extension does not generalize classical truth and falsehood in the case in which $Q=\neg P$, while that given by (1)-(2) does.

Before we apply this extension to the different combinations of fuzziness and bipolarity types, we enunciate the following theorem, that states the properties verified by such an extension for the case $L^{+}=L^{-}=L^{I}$, i.e., in a simultaneous B2 and F2 situation in which imprecision is allowed for the couple $\left(\mu^{+}, \mu^{-}\right)$:

Theorem 1: Let $\mu^{+}=\left[\mu_{L}^{+}, \mu_{U}^{+}\right]$and $\mu^{-}=\left[\mu_{L}^{-}, \mu_{U}^{-}\right] \quad\left(0 \leq \mu_{L}^{-} \leq \mu_{U}^{-} \leq 1\right.$, $\left.0 \leq \mu_{L}^{+} \leq \mu_{U}^{+} \leq 1\right)$ be the positive and negative interval-valued evaluations of a predicate " $x \in P$ ". Let also $t=\left[t_{L}, t_{U}\right], f=\left[f_{L}, f_{U}\right], i=\left[i_{L}, i_{U}\right]$ and $k=\left[k_{L}, k_{U}\right]$ be the 4-valued extension associated to the pair $\left(\mu^{+}, \mu^{-}\right)$, obtained through the formulae (5)-(8). Then $k_{L}$ and $i_{U}$ as well as $i_{L}$ and $k_{U}$ are mutually exclusive and it holds that $t_{L}+f_{L}+i_{U}+k_{U}=t_{U}+f_{U}+i_{L}+k_{L}=1+m$, where $m=\min \left\{u^{+}, u^{-}, u_{t}, u_{f}, u_{u}, u_{k}\right\} \quad$ and $u^{+}=\mu_{U}^{+}-\mu_{L}^{+}, u^{-}=\mu_{U}^{-}-\mu_{L}^{-}, u_{t}=t_{U}-t_{L}, \quad u_{f}=f_{U}-f_{L}, \quad u_{i}=i_{U}-i_{L}, u_{k}=k_{U}-k_{L}$ are the uncertainty degrees of each interval.

Proof: Let us denote $M_{1}=t_{L}+f_{L}+i_{U}+k_{U}, \quad M_{2}=t_{U}+f_{U}+i_{L}+k_{L}$, $s^{U}=\mu_{U}^{+}+\mu_{U}^{-}, s^{L}=\mu_{L}^{+}+\mu_{L}^{-}, s_{1}=\mu_{L}^{+}+\mu_{U}^{-}, s_{2}=\mu_{U}^{+}+\mu_{L}^{-}$and recall that

$t_{L}=\mu_{L}^{+} \wedge\left(1-\mu_{U}^{-}\right), t_{U}=\mu_{U}^{+} \wedge\left(1-\mu_{L}^{-}\right), f_{L}=\mu_{L}^{-} \wedge\left(1-\mu_{U}^{+}\right), f_{U}=\mu_{U}^{-} \wedge\left(1-\mu_{L}^{+}\right)$

$i_{L}=\left(1-\mu_{U}^{+}-\mu_{U}^{-}\right) \vee 0, i_{U}=\left(1-\mu_{L}^{+}-\mu_{L}^{-}\right) \vee 0, k_{L}=\left(\mu_{L}^{+}+\mu_{L}^{-}-1\right) \vee 0, k_{U}=\left(\mu_{U}^{+}+\mu_{U}^{-}-1\right) \vee 0$.

Clearly, both $k_{L}, i_{U}$ and $i_{L}, k_{U}$ are mutually exclusive. Then notice that $s_{1}$ determines the relation between $\mu_{L}^{+}$and $1-\mu_{U}^{-}$, since $s_{1}<1$ (resp. $s_{1} \geq 1$ ) implies $\mu_{L}^{+}<1-\mu_{U}^{-}\left(\mu_{L}^{+} \geq 1-\mu_{U}^{-}\right)$and therefore $t_{L}=\mu_{L}^{+}$and $f_{U}=\mu_{U}^{-}\left(t_{L}=1-\mu_{U}^{-}\right.$and $f_{U}=1-\mu_{L}^{+}$). Similarly, $s_{2}$ determines the values $t_{U}$ and $f_{L}$, and thus the 4 possible situations of $s_{1}$ and $s_{2}$ wrt 1 produce four possible cases for $t_{L}, t_{U}, f_{L}, f_{U}$.

On the other hand, $s^{U}$ and $s^{L}$ determine, respectively, the values $i_{L}, k_{U}$ and $i_{U}, k_{L}$ (e.g., $s^{U}<1$ implies $i_{L}=1-s^{U}$ and $k_{U}=0$ ), but only 3 cases are now possible since the case $s^{U}<1, s^{L} \geq 1$ is excluded as it is $s^{L} \leq s^{U}$. Moreover, from the $12(4 \cdot 3)$ cases in principle possible, 6 have to be removed since the constraints 
On the semantics of bipolarity and fuzziness

$0 \leq s^{L} \leq s_{1}, s_{2} \leq s^{U} \leq 2$ and $s^{L}+s^{U}=s_{1}+s_{2}$ also hold. Thus, this proof will consist on showing that the equality $M_{1}=M_{2}=1+m$ holds in each of these 6 cases:

1) $s_{1}, s_{2}, s^{L}, s^{U}<1$ : in this case it holds that $M_{1}=\mu_{L}^{+}+\mu_{L}^{-}+1-\mu_{L}^{+}-\mu_{L}^{-}=1$, $M_{2}=\mu_{U}^{+}+\mu_{U}^{-}+1-\mu_{U}^{+}-\mu_{U}^{-}=1, u_{t}=u^{+}, u_{f}=u^{-}, u_{i}=u^{+}+u^{-}, u_{k}=0$, hence $m=0$ and $M_{1}=M_{2}=1+m$.

2) $s_{1}, s_{2}, s^{L}<1, s^{U} \geq 1$ : now it is $M_{1}=\mu_{L}^{+}+\mu_{L}^{-}+1-\mu_{L}^{+}-\mu_{L}^{-}+\mu_{U}^{+}+\mu_{U}^{-}-1=$ $=\mu_{U}^{+}+\mu_{U}^{-}=s^{U}, M_{2}=\mu_{U}^{+}+\mu_{U}^{-}=s^{U}, u_{t}=u^{+}, u_{f}=u^{-}, u_{i}=1-s^{L}, u_{k}=s^{U}-1$, and since $u^{+}-\left(s^{U}-1\right)=1-s_{1}>0, u^{-}-\left(s^{U}-1\right)=1-s_{2}>0,1-s^{L}-\left(s^{U}-1\right)=$ $=2-\left(s^{L}+s^{U}\right)=2-\left(s_{1}+s_{2}\right)>0$, then $m=u_{k}=s^{U}-1$ and $M_{1}=M_{2}=1+m$.

3) $s_{1}, s^{L}<1, s_{2}, s^{U} \geq 1$ : it is $M_{1}=\mu_{L}^{+}+1-\mu_{U}^{+}+1-\mu_{L}^{+}-\mu_{L}^{-}+\mu_{U}^{+}+\mu_{U}^{-}-1=$ $1+\mu_{U}^{-}-\mu_{L}^{-}=1+u^{-}, M_{2}=1-\mu_{L}^{-}+\mu_{U}^{-}=1+u^{-}, u_{t}=1-s^{L}, u_{f}=s^{U}-1$, $u_{i}=s^{U}-1, \quad u_{k}=1-s^{L}, \quad$ and it also holds that $u^{+}-u^{-}=s_{2}-s_{1}>0$, $1-s^{L}-u^{-}=1-s_{1}>0, s^{U}-1-u^{-}=s_{2}-1 \geq 0$, so $m=u^{-}$and $M_{1}=M_{2}=1+m$ again.

4) $s_{2}, s^{L}<1, s_{1}, s^{U} \geq 1$ : it is $M_{1}=1-\mu_{U}^{-}+\mu_{L}^{-}+1-\mu_{L}^{+}-\mu_{L}^{-}+\mu_{U}^{+}+\mu_{U}^{-}-1=$ $1+\mu_{U}^{+}-\mu_{L}^{+}=1+u^{+}, M_{2}=\mu_{U}^{+}+1-\mu_{L}^{+}=1+u^{+}, u_{t}=s^{U}-1, u_{f}=1-s^{L}, u_{i}=1-s^{L}$, $u_{k}=s^{U}-1$. As $u^{-}-u^{+}=s_{1}-s_{2}>0, \quad 1-s^{L}-u^{+}=1-s_{2}>0, \quad s^{U}-1-u^{+}=$ $s_{1}-1 \geq 0$, it is $m=u^{+}$and $M_{1}=M_{2}=1+m$.

5) $s^{L}<1, s_{1}, s_{2}, s^{U} \geq 1$ : it is $M_{1}=1-\mu_{U}^{-}+1-\mu_{U}^{+}+1-\mu_{L}^{+}-\mu_{L}^{-}+\mu_{U}^{+}+\mu_{U}^{-}-1=$ $2-\mu_{L}^{+}-\mu_{L}^{-}=1+\left(1-s^{L}\right), M_{U}=1-\mu_{L}^{+}+1-\mu_{L}^{-}=1+\left(1-s^{L}\right), u_{t}=u^{-}, u_{f}=u^{+}, u_{i}=1-s^{L}$, $u_{k}=s^{U}-1, \quad$ and also $u^{+}-\left(1-s^{L}\right)=s_{1}-1 \geq 0, \quad u^{-}-\left(1-s^{L}\right)=s_{2}-1 \geq 0$, $s^{U}-1-\left(1-s^{L}\right)=s^{U}+s^{L}-2=s_{1}+s_{2}-2 \geq 0$, thus $m=1-s^{L}$ and $M_{1}=M_{2}=1+m$.

6) $s_{1}, s_{2}, s^{L}, s^{U} \geq 1$ : finally, it is $M_{1}=1-\mu_{U}^{-}+1-\mu_{U}^{+}+\mu_{U}^{+}+\mu_{U}^{-}-1=1$, $M_{2}=1-\mu_{L}^{+}+1-\mu_{L}^{-}+\mu_{L}^{+}+\mu_{L}^{-}-1=1, u_{t}=u^{-}, u_{f}=u^{+}, u_{i}=0, u_{k}=u^{+}+u^{-}$, so it follows $m=u_{i}=0$ and $M_{1}=M_{2}=1+m$.

Next, Theorem 1 will be applied to different combinations of bipolarity and fuzziness types to illustrate its particular semantics:

Type-1 fuzziness and bipolarity (F1,B1)

In this case, $L=[0,1]$ and $Q=\neg P, \quad$ so it is $\mu^{+}=\mu_{P}$ and $\mu^{-}=\mu_{\neg P}=n\left(\mu_{P}\right)=1-\mu_{P}$. This leads to $t=\mu^{+}, f=\mu^{-}$and $k=i=0$. Particularly, $m=0$ as uncertainty regarding the actual values of $\mu^{+}, \mu^{-}$is not allowed. For 
example, let us suppose $x \in X$ such that $\mu_{P}(x)=0.6$. Then we get the evidence couple $\left(\mu^{+}, \mu^{-}\right)=(0.6,0.4)$ and the evidence matrix

$$
E M_{1}=\left[\begin{array}{cc}
0.6 & 0 \\
0 & 0.4
\end{array}\right] .
$$

Thus, the extension (4)-(5) generalizes fuzzy logic truth and falsehood (identified with $\mu_{P}$ and $\mu_{P}$ ), and reflects the $\mathrm{B} 1$ semantics of this setting, that excludes $k$ and $i$.

Type-2 fuzziness and type-1 bipolarity (F2,B1)

Suppose now that in the previous example, the value $\mu_{P}(x)$ is not precisely known, but rather it is known that such a value lies in the interval $\left[\mu_{L}(x), \mu_{U}(x)\right]_{P}$. Thus, it still is assumed $Q=\neg P$, but now $L=L^{I}$ and then $\mu^{+}=\left[\mu_{L}, \mu_{\mathrm{U}}\right]_{P}$ and $\mu^{-}=n_{L^{I}}\left(\left[\mu_{L}, \mu_{U}\right]_{P}\right)=\left[1-\mu_{U}, 1-\mu_{L}\right]_{\neg P}$. Thus, it is again obtained $t=\mu^{+}$and $f=\mu^{-}$but now $k=i=[0, u]$ and $m=u$, with $u=\mu_{U}-\mu_{L}$ the uncertainty associated to $\mu_{P}$. For example, if $\mu^{+}=[0.5,0.7]$ (and thus $\mu^{-}=[0.3,0.5]$ ), the matrix

$$
E M_{2}=\left[\begin{array}{cc}
{[0.5,0.7]} & {[0,0.2]} \\
{[0,0.2]} & {[0.3,0.5]}
\end{array}\right]
$$

is obtained, showing that now the evaluations of positive truth $t$ and falsehood $f$ are also uncertain, allowing $i$ and $k$ to be non-zero. However, if uncertainty is reduced, i.e. as $u$ tends to $0, k$ and $i$ tend to $[0,0]$, approaching the case $(\mathrm{F} 1, \mathrm{~B} 1)$.

Type-1 fuzziness and type-2 bipolarity $(\mathrm{F} 1, \mathrm{~B} 2)$

If negative information about " $x \in P$ " is allowed to be independent from the negation $n$ in the first case $(\mathrm{F} 1, \mathrm{~B} 1)$, then we obtain a B2 framework in which $Q \neq \neg P$, $\mu^{+}=\mu_{P}, \mu^{-}=\mu_{Q}$ and $L^{+}=L^{-}=L=[0,1]$. Thus, $m=u=0$, but $t(f)$ can no more be identified with $\mu^{+}\left(\mu^{-}\right)$, and one of the states $i, k$ can hold. For example, if $\left(\mu^{+}, \mu^{-}\right)=(0.6,0.7)$, we get the matrix

$$
E M_{3}=\left[\begin{array}{cc}
0.3 & 0 \\
0.3 & 0.4
\end{array}\right],
$$

which shows that $t \neq \mu^{+}, f \neq \mu^{-}$and informs that information is partially conflictive ( $k=0.3)$, thus entailing $t<\mu^{+}$and $f<\mu^{-}$.

Similarly, notice that $\left(\mu^{+}, \mu^{-}\right)=(0.5,0.3) \in L^{*}$ is the evidence couple associated

to the interval $[0.5,0.7] \in L^{I}$ of the previous case by means of the isomorphism $\Phi: L^{*} \rightarrow L^{I}$ (see Section 3), and it produces the evidence matrix

$$
E M_{4}=\left[\begin{array}{cc}
0.5 & 0.2 \\
0 & 0.3
\end{array}\right] \text {. }
$$

It is $E M_{2} \neq E M_{4}$, and not only because $E M_{2}$ does introduce uncertainty while $E M_{4}$ does not. If uncertainty $u$ is reduced, then it could be $\mu_{E M_{2}}^{+}=[0.5,0.5]$, in such a way 
On the semantics of bipolarity and fuzziness

that $t_{E M_{2}}=[0.5,0.5]=0.5=t_{E M_{4}}$, but then it is $f_{E M_{2}}=[0.5,0.5] \neq 0.3=f_{E M_{4}}$ and $i_{E M_{2}}=[0,0] \neq 0.2=i_{E M_{4}}$ as $\mu_{E M_{2}}^{-}=n_{L^{I}}\left(\mu_{E M_{2}}^{+}\right)=[0.5,0.5]$ due to the B1 setting of IVFS. However, BAFS allows $\mu^{-}$to be independent from $\mu^{+}$since they exhibit a type-2 bipolarity, though the condition $\mu^{+}+\mu^{-} \leq 1$ entails that $k=0$. Thus, it is clear that semantics of BAFS is different from that of IVFS, and their equivalence should be regarded as a formal coincidence between two scales that belong to different universes, since $[0,1] \subset L^{I} \subset F([0,1])$ while $L^{*} \subset[0,1]^{2} \subset L^{I} \times L^{I} \subset F([0,1]) \times F([0,1])$.

Type-2 fuzziness and bipolarity (F2,B2)

Finally, suppose that uncertainty about $\mu^{+}, \mu^{-}$is allowed in a B2 setting, in such a way that an evidence couple $\left(\mu^{+}, \mu^{-}\right) \in L^{I} \times L^{I}$ is obtained. Notice that this case generalizes all the previous ones and corresponds to the hypothesis of Theorem 1 . Thus, if $\mu^{+}=[0.5,0.7]$ and $\mu^{-}=[0.6,0.9]$, we get the evidence matrix

$$
E M_{5}=\left[\begin{array}{cc}
{[0.1,0.4]} & {[0,0]} \\
{[0.1,0.6]} & {[0.3,0.5]}
\end{array}\right],
$$

and notice that now each of its elements have a different associated uncertainty (and $m=0$ ) and that these results extend those of $E M_{1}, E M_{2}$ and $E M_{3}$. Note also that either $u^{+}$or $u^{-}$can be reduced without affecting the other, and in the limit case in which $u^{+}=u^{-}=0$, a F1 and B2 setting is reached. Instances of B2 and F2 objects are Atanassov IVFS (AIVFS, see [2]), though the same argument of Section 3 applies in order to affirm that they can not be seen as B2 objects unless it is assumed the existence of a negative pole $Q \neq \neg P$ (leading to bipolar AIVFS).

\section{Conclusions}

The notions of fuzziness and bipolarity have been revised, and its relations analyzed. As these notions were developed to address different kinds of uncertainty, respectively linguistic imprecision and information relevance and polarity, both of them are in fact complementary and independent. An F2 object could be B2 or not, and reciprocally a B2 formalism could allow uncertainty (thus being also a F2 object) or not. Particularly, in this paper these differences have become formally evident, clarifying some aspects of the relations between $\mathrm{B} 1, \mathrm{~F} 2$ formalisms and B2,F1 ones, and hopefully allowing to lead to an at least relative solution over the controversy between Atanassov's supporters and critics. As this paper implicitly shows, there is a difference between intuitionism (in the sense of using a sub-additive negation $n(\mu) \leq 1-\mu$ ) and bipolarity. However, if $Q \neq \neg P$ is assumed, then BAFS and BAIVFS have been shown to be relevant $\mathrm{B} 2$ objects. Table 1 presents a summary of the cases and formalisms analyzed in this paper, which have been shown to exhibit a different semantics in terms of fourvalued extensions, and thus in terms of its ability to model knowledge states. 
Table 1. Combinations of bipolarity and fuzziness types- 1 and 2 .

\begin{tabular}{|c|l|l|}
\hline Bipolarity & Type-1 (F1) & Type-2 (F2) \\
\hline Type-1 (B1) & T1FS & T2FS, IVFS \\
\hline Type-2 (B2) & BAFS, BFS & BT2FS, BAIVFS \\
\hline
\end{tabular}

Further work is forthcoming in order to extend this work in several directions: lattices (see [5] and notice that all formalisms in Table 1 are special cases of L-fuzzy sets [16]) will be introduced in order to obtain a more rigorous and general logical and formal setting. The relations between the notions of bipolarity and intuitionism, the use of a sub-additive negation and dissimilarity operators [31] will be further explored (see [15]), as well as the possible use of a F2 formalism in order to combine fuzziness and other types of uncertainty theories.

\section{Acknowledgments}

This research has been partially supported by grant TIN2009-07901.

\section{References}

[1] Atanassov KT (1986) Intuitionistic Fuzzy-Sets, Fuzzy Set Syst, 20 (1) 87-96

[2] Atanassov KT, Gargov G (1989) Interval Valued Intuitionistic Fuzzy Sets,F Set Syst, 31(3) 343-349

[3] Atanassov KT (2005) Answer to D. Dubois, S. Gottwald, P. Hajek, J. Kacprzyk and H. Prade's paper "Terminological difficulties in fuzzy set theory - the case of "Intuitionistic fuzzy sets", Fuzzy Set Syst, 156 (3) 496-499

[4] Beringer J, Hullermeier E (2008) Case-based learning in a bipolar possibilistic framework, Int J Intell Syst, 23 (10) 1119-1134

[5] Birkhoff G (1948) Lattice theory, Amer. Math. Soc. Colloq. Publ. vol. 25, New York

[6] Bustince H, Pagola M, Barrenechea E, Fernandez J, Melo-Pinto P, Couto P, Tizhoosh HR, Montero J (2010) Ignorance functions. An application to the calculation of the threshold in prostate ultrasound images, Fuzzy Set Syst, 161 (1) 20-36

[7] Cacioppo JT, GardnerWL, Berntson GG (1997) Beyond bipolar conceptualizations and measures: The case of attitudes and evaluative space. Pers Soc Psychol Rev 1:3-25.

[8] Cornelis C, Atanassov K, Kerre E, Intuitionistic fuzzy sets and interval-valued fuzzy sets: a critical comparison, Proc. Eusflat'03, 2003, 159-163

[9] Deschrijver G, Kerre E (2004) On the relationship between some extensions of fuzzy set theory, Fuzzy Set Syst 133 227-235.

[10] Dubois D, Gottwald S, Hajek P, Kacprzyk J, Prade H (2005) Terminological difficulties in fuzzy set theory - The case of "Intuitionistic Fuzzy Sets", Fuzzy Set Syst, 156 (3) 485-491

[11] Dubois D, Prade H (2008) Special issue on bipolar representations of information and preference part 1A: Cognition and decision - Foreword, Int J Intell Syst, 23 (8) 863-865

[12] Dubois D, Prade H (2008) An introduction to bipolar representations of information and preference, Int J Intell Syst, 23 (8) 866-877 
[13] Dubois D, Prade H (2009) An overview of the asymmetric bipolar representation of positive and negative information in possibility theory, Fuzzy Set Syst, 160 (10) 1355-1366

[14] Fodor J, Roubens M (1994) Fuzzy preference modelling and multicriteria decision support, Kluwer Academic, Dordrecht ; Boston

[15] Franco CA, Montero J, Rodriguez JT (2011) Aggregation Weights for a Fuzzy PreferenceAversion Model, Procs. of the WConSC'11, 199-206

[16] Goguen JA (1967) L-fuzzy sets, J. Math. Anal. Appl, 18 145-174

[17] Grabisch M, Labreuche C (2008) A decade of application of the Choquet and Sugeno integrals in multi-criteria decision aid. A Quarterly Journal of Operations Research 6, 1-44.

[18] Grattan-Guinness I (1975) Fuzzy membership mapped onto interval and many-valued quantities, Z. Math. Logik Grundlag. Mathe., 22 149-160

[19] Hullermeier E (2005) Fuzzy methods in machine learning and data mining: Status and prospects, Fuzzy Set Syst, 156 (3) 387-406

[20] Kacprzyk J, Zadrozny S (2010) Soft computing and web intelligence for supporting consensus reaching, Soft Computing, 14 (8) 833-846

[21] Kahneman D, Tversky A. (1979) Prospect theory: decision analysis under risk. Econom. 47, 263-291

[22] Klir GJ, Yuan B (1995) Fuzzy Sets and Fuzzy Logic: Theory and Applications. Englewood Cliffs, NJ: Prentice-Hall

[23] Labreuche C, Grabisch M (2007) The representation of conditional relative importance between criteria. Annals of Operations Research 154, 93-122.

[24] Mamdani EH (1974) Application of Fuzzy Algorithms for the Control of a Dynamic Plant, Proc. IEE, 121 (12): 1585-1588

[25] Medin D, Schwanenflugel P (1981) Linear separability in classification learning, J. of Experimental Psychology: Human Learning \& Memory, 7 355-368

[26] Montero J (1987) Arrow's theorem under fuzzy rationality. Behavioral Science 32, 267-273.

[27] Montero J, Gómez D, Bustince H (2007) On the relevance of some families of fuzzy sets, Fuzzy Set Syst, 158 (22): 2439-2442

[28] Osgood CE, Suci GJ, Tannenbaum PH (1957) The measurement of meaning. Chicago, UIP

[29] Öztürk M, Tsoukiàs A (2007) Modelling uncertain positive and negative reasons in decision aiding, Decision Support Systems, 43 (4): 1512-1526

[30] Rodriguez JT, Vitoriano B, Montero J (2011) Rule-based classification by means of bipolar criteria, IEEE MCDM 2011, 197-204

[31] Rodriguez JT, Franco CA, Vitoriano B, Montero J (2011) An axiomatic approach to the notion of semantic antagonism, Procs. of the IFSA-AFSS'11, FT104-1/6

[32] Roy B (1968) Ranking and Choice in Pace of Multiple Points of View (Electre Method), Revue Francaise D Informatique De Recherche Operationnelle, 2(8) 57-\&

[33] Schweizer B, Sklar A (1983) Probabilistic Metric Spaces. North-Holland/Elsevier, New York.

[34] Trillas E (2006) On the use of words and fuzzy sets, Information Sciences, 176 (11) 14631487 
[35] Tsoukiàs A (2002) A first-order, four valued, weakly paraconsistent logic and its relation to rough sets semantics, Foundations of Computing and Decision Sciences, 12 85-108

[36] Turunen E, Ozturk M, Tsoukiàs A (2010) Paraconsistent semantics for Pavelka style fuzzy sentential logic Fuzzy Set Syst, 161 (14) 1926-1940

[37] Tversky A, Kahneman D (1992) Advances in prospect theory: cumulative representation of uncertainty. Journal of Risk and Uncertainty 5, 297-323

[38] Victor P, Cornelis C, Cock M D, Teredesai A (2009) Trust- and distrust-based recommendations for controversial reviews, Proc. of WEBSCI09

[39] Zadeh LA (1965) Fuzzy Sets, Information and Control, 8 (3) 338-353

[40] Zadeh, LA (1975) The Concept of a Linguistic Variable and Its Application to Approximate Reasoning, Information Sciences 9 (1) 43-80 